Communications in Nonlinear Science and Numerical Simulation 11 (2006) 885-898

\title{
Fractional dynamics of systems with long-range interaction
}

\author{
Vasily E. Tarasov ${ }^{1,2}$ and George M. Zaslavsky ${ }^{2,3}$ \\ 1) Skobeltsyn Institute of Nuclear Physics, \\ Moscow State University, Moscow 119992, Russia \\ 2) Courant Institute of Mathematical Sciences, New York University \\ 251 Mercer Street, New York, NY 10012, USA, \\ 3) Department of Physics, New York University, \\ 2-4 Washington Place, New York, NY 10003, USA
}

\begin{abstract}
We consider one-dimensional chain of coupled linear and nonlinear oscillators with long-range power wise interaction defined by a term proportional to $1 / \mid n-$ $\left.m\right|^{\alpha+1}$. Continuous medium equation for this system can be obtained in the socalled infrared limit when the wave number tends to zero. We construct a transform operator that maps the system of large number of ordinary differential equations of motion of the particles into a partial differential equation with the Riesz fractional derivative of order $\alpha$, when $0<\alpha<2$. Few models of coupled oscillators are considered and their synchronized states and localized structures are discussed in details. Particularly, we discuss some solutions of time-dependent fractional Ginzburg-Landau (or nonlinear Schrodinger) equation.
\end{abstract}

PACS: 05.45.-a; 45.05.+x; 45.50.-j

Keywords: Long-range interaction, Fractional oscillator, Synchronization, Fractional GinzburgLandau equation 


\section{Introduction}

Although the fractional calculus is known for more than two hundred years and its developing is an active area of mathematics, appearance and use of it in physical literature is fairly recent and sometimes is considered as exotic. In fact, there are many different areas where fractional equations, i.e., equations with fractional integro-differentiation, describe real processes. Between the most related areas are chaotic dynamics [1, random walk in fractal space-time [2] and random processes of Levy type [3, 4, 5, 6]. The physical reasons for the appearance of fractional equations are intermittancy, dissipation, wave propagation in complex media, long memory, and others. An important area of the application of long-range interaction includes collective oscillation and synchronization in physics, chemistry, biology, and neuroscience actively studied recently [7, 8, 9]. Beginning from the pioneering contributions by Winfree [10] and Kuramoto [11, studies of synchronization in populations of coupled oscillators becomes an active field of research in biology and chemistry. The synchronization can also be considered in complex oscillatory medium, where each site (element) performs self-sustained oscillations. A good physical and chemical example is the oscillatory Belousov-Zhabotinsky reaction [12, 13, 11] in a medium where different sites can oscillate with different periods and phases.

Complex Ginzburg-Landau equation is canonical model for oscillatory systems with local coupling near Hopf bifurcation. Recently, Tanaka and Kuramoto [14 have shown how, in the vicinity of the bifurcation, the description of an array of nonlocally coupled oscillators can be reduced to the complex Ginzburg-Landau equation. In Ref. [15], a model of population of diffusively coupled oscillators with limit-cycles is described by the complex Ginzburg-Landau equation with nonlocal interaction.

Nonlocal coupling means interaction with oscillators or other objects distanced arbitrary far from each others. The corresponding interaction potential could be with a characteristic length Refs. [16, 17, 15] or without it. The long-range interaction that decreases as $1 /|x|^{\alpha+1}$ with $0<\alpha<2$ was considered in Refs. [18, 19, 20, 21, 22] with respect to the system's thermodynamics and phase transition. It is also shown in 23] that using the Fourier transform and limit for the wave number $k \rightarrow 0$, the long-range term interaction leads under special conditions to the fractional dynamics.

In the last decade it is found that many physical processes can be adequately described by 
equations that consist of derivatives of fractional order. In a fairly short period of time the list of such applications becomes long and the area of applications is broad. Even in a concise form, the applications include material sciences [24, 25, 26, 27], chaotic dynamics [1], quantum theory [28, 29, 30], physical kinetics [1, 3, 32, 31], fluids and plasma physics [33, 34], and many others physical topics related to wave propagation [35], long-range dissipation [36], anomalous diffusion and transport theory (see reviews [1, 2, 24, 4, 37]). Particularly, fractional Ginzburg-Landau equation was suggested in [38, 39, 40].

In this paper we would like to strength the state that the one-dimentional chain of particles with long-range power type interaction can be asymptotically described by the continuous medium equation with fractional space derivative. We introduce, under some conditions, a corresponding transform operator that map the system of large number ordinary differential equations of particles motion into the equation with partial and fractional derivatives. It is worthwhile to mention that possibility of such an operation has an immediate physical consequence: fractional dynamics equation has solutions that describe coherent structures with power-like tails.

In Section 2, we introduce the transform operator. In Section 3, some particular solutions are derived with a constant wave number for the fractional Ginzburg-Landau (FGL) equation that can be interpreted as synchronization in the oscillatory medium. In Section 4, we consider forced FGL equation for isochronous case. In Section 5, we derive solutions of the fractional GinzburgLandau equation near a limit cycle. These solutions are interpreted as coherent structures in the oscillatory medium with long-range interaction.

\section{Long-range interaction of oscillators}

Consider a system with Hamiltonian

$$
H=\sum_{n=-\infty}^{+\infty}\left[\frac{1}{2} \dot{u}_{n}^{2}+\frac{1}{2} g_{0} \sum_{\substack{m=-\infty \\ m \neq n}}^{+\infty} \frac{1}{|n-m|^{1+\alpha}} u_{n} u_{m}+V\left(u_{n}\right)\right]
$$

where $g_{0}$ and $\alpha$ are some constants, and $u_{n}=u_{n}(t)$. The corresponding equations of motion are

$$
\frac{\partial^{2} u_{n}}{\partial t^{2}}+g_{0} \sum_{\substack{m=-\infty \\ m \neq n}}^{+\infty} \frac{1}{|n-m|^{1+\alpha}} u_{m}+V^{\prime}\left(u_{n}\right)=0
$$


with $V^{\prime}(u)=\partial V(u) / \partial u$. Let us introduce notations

$$
H_{i n t} \equiv \frac{1}{2} g_{0} \sum_{\substack{m=-\infty \\ m \neq n}}^{+\infty}|n-m|^{-(1+\alpha)} u_{n} u_{m}
$$

and

$$
\hat{T}_{\alpha} u_{n} \equiv \sum_{\substack{m=-\infty \\ m \neq n}}^{+\infty}|n-m|^{-(1+\alpha)} u_{m}
$$

The standard transformation from the set of equations (2) to the continuous medium equations is in replacements

$$
u_{n}(t) \equiv u\left(x_{n}, t\right) \rightarrow u(x, t), \quad x_{n}=n \Delta x,
$$

where $n$ is a positive integer number, $\Delta x$ is a distance between particles, and the limit $\Delta x \rightarrow 0$ is applied.

A specific form of $H_{\text {int }}$ and the corresponding term $\hat{T}_{\alpha}$ in the equation of motion (2) create a possibility to present the continuous medium equations in a form that consists of fractional derivatives. For this goal, we use the notation

$$
\begin{gathered}
u(x, t)=\frac{1}{2 \pi} \int_{-\infty}^{+\infty} d k \tilde{u}(k, t) e^{i k x} \equiv \mathcal{F}^{-1}\{\tilde{u}(k, t)\} \\
\tilde{u}(k, t)=\int_{-\infty}^{+\infty} d x Z(x, t) e^{-i k x} \equiv \mathcal{F}\{u(x, t)\}
\end{gathered}
$$

where $\mathcal{F}\{u(x, t)\}$ is Fourier transform of $u(x, t)$ with respect to $x$. Then, replacing (5) in (2), we obtain $\hat{T}_{\alpha} u_{n}(t) \rightarrow \hat{T}_{\alpha} u(x, t)$, and after applying Fourier transform to (2),

$$
\frac{\partial^{2} u(k, t)}{\partial t^{2}}+g_{0}\left[T_{\alpha}(k)-T_{\alpha}(0)\right] u(k, t)+\mathcal{F}\left\{V^{\prime}(u(x, t))\right\}=0
$$

where

$$
T_{\alpha}(k)=2 \Gamma(-\alpha) \cos (\pi \alpha / 2)|k|^{\alpha}+2 \sum_{n=0}^{\infty} \frac{\zeta(\alpha+1-2 n)}{(2 n) !}\left(-k^{2}\right)^{n}, \quad|k|<1, \quad \alpha \neq 0,1,2,3 \ldots
$$

and $T_{\alpha}(0)=2 \zeta(1+\alpha)$ and $\zeta$ is the Riemann zeta-function. Function $T_{\alpha}(k)$ can also be presented in the form

$$
T_{\alpha}(k)=\sum_{\substack{n=-\infty \\ n \neq 0}}^{+\infty} e^{-i k n} \frac{1}{|n|^{\alpha+1}}=2 \sum_{n=1}^{\infty} \frac{\cos (k n)}{n^{1+\alpha}}=L i_{\alpha+1}\left(e^{i k}\right)+L i_{\alpha+1}\left(e^{-i k}\right)
$$


where $L i_{\alpha}(z)$ is a polylogarithm function [46]

$$
L i_{\beta}\left(e^{z}\right)=\Gamma(1-\beta)(-z)^{\beta-1}+\sum_{n=0}^{\infty} \frac{\zeta(\beta-n)}{n !} z^{n}, \quad|z|<2 \pi, \quad \beta \neq 1,2,3 \ldots
$$

After substitution (8) into (7) obtain

$$
\frac{\partial^{2} \tilde{u}(k, t)}{\partial t^{2}}=\mathcal{F}\left\{V^{\prime}(u(x, t))\right\}-g_{0} a_{\alpha}|k|^{\alpha} \tilde{u}(k, t)-2 g_{0} \sum_{n=1}^{\infty} \frac{\zeta(\alpha+1-2 n)}{(2 n) !}\left(-k^{2}\right)^{n} \tilde{u}(k, t),
$$

where

$$
a_{\alpha}=2 \Gamma(-\alpha) \cos (\pi \alpha / 2), \quad \alpha \neq 0,1,2, \ldots
$$

From Eq. (11) we obtain the equation for the field $u(x, t)$ using the definition (6)

$$
\frac{\partial^{2} u(x, t)}{\partial t^{2}}=V^{\prime}(u(x, t))+g_{0} a_{\alpha} \frac{\partial^{\alpha}}{\partial|x|^{\alpha}} u(x, t)-2 g_{0} \sum_{n=1}^{\infty} \frac{\zeta(\alpha+1-2 n)}{(2 n) !} \frac{\partial^{2 n}}{\partial x^{2 n}} u(x, t), \quad(\alpha \neq 0,1,2 \ldots) .
$$

Here, we use the connection between Riesz fractional derivative and its Fourier transform [43]:

$$
|k|^{\alpha} \longleftrightarrow-\frac{\partial^{\alpha}}{\partial|x|^{\alpha}}, \quad k^{2} \longleftrightarrow-\frac{\partial^{2}}{\partial|x|^{2}}
$$

Such construction that leads to the equation with fractional derivative appears also in [23].

The properties of the Riesz derivative can be found in [43, 44, 45]. Its another expression is

$$
\frac{\partial^{\alpha}}{\partial|x|^{\alpha}} u(x, t)=-\frac{1}{2 \cos (\pi \alpha / 2)}\left(\mathcal{D}_{+}^{\alpha} u(x, t)+\mathcal{D}_{-}^{\alpha} u(x, t)\right)
$$

where $\alpha \neq 1,3,5 \ldots$, and $\mathcal{D}_{ \pm}^{\alpha}$ are Riemann-Liouville left and right fractional derivatives

$$
\begin{aligned}
& \mathcal{D}_{+}^{\alpha} u(x, t)=\frac{1}{\Gamma(n-\alpha)} \frac{\partial^{n}}{\partial x^{n}} \int_{-\infty}^{x} \frac{u(\xi, t) d \xi}{(x-\xi)^{\alpha-n+1}} \\
& \mathcal{D}_{-}^{\alpha} u(x, t)=\frac{(-1)^{n}}{\Gamma(n-\alpha)} \frac{\partial^{n}}{\partial x^{n}} \int_{x}^{\infty} \frac{u(\xi, t) d \xi}{(\xi-x)^{\alpha-n+1}}
\end{aligned}
$$

where $n-1<\alpha<n$.

The obtained expressions (11) and (13) can be simplified in the so-called infrared approximation $k \rightarrow 0$. Then Eq. (11) can be rewritten as

$$
\frac{\partial}{\partial t} \tilde{u}(k, t)=\mathcal{F}\left\{V^{\prime}(u(x, t))\right\}+g_{0} \mathcal{T}_{\alpha}(k) \tilde{u}(k, t), \quad k \rightarrow 0, \quad(1<\alpha<3),
$$

where we use $\mathcal{T}_{\alpha}(k)$ as an approximation expression for $T_{\alpha}(k)$ :

$$
\mathcal{T}_{\alpha}(k)=2 \Gamma(-\alpha) \cos (\pi \alpha / 2)|k|^{\alpha}-\zeta(\alpha-1) k^{2}, \quad(1<\alpha<2)
$$




$$
\begin{gathered}
\mathcal{T}_{2}(k)=\text { const } \ln k^{2}, \quad(\alpha=2), \\
\mathcal{T}_{\alpha}(k)=J_{0} k^{2}, \quad(\alpha>2)
\end{gathered}
$$

The expression for $\mathcal{T}_{\alpha}(k)$ can be considered as a Fourier transform of an operator $\hat{\mathcal{T}}_{\alpha}(x)$ :

$$
\hat{\mathcal{T}}_{\alpha}(x)=\mathcal{F}^{-1}\left\{\mathcal{T}_{\alpha}(k)\right\}=a_{\alpha} \frac{\partial^{\alpha}}{\partial|x|^{\alpha}}+\zeta(\alpha-1) \frac{\partial^{2}}{\partial x^{2}} .
$$

Applying inverse Fourier transform to (17), we also obtain

$$
\frac{\partial^{2}}{\partial t^{2}} u(x, t)=V^{\prime}(u(x, t))+g_{0} \hat{\mathcal{T}}_{\alpha}(x) u(x, t), \quad(1<\alpha<2) .
$$

This equation can be considered as an equation for continuous oscillatory medium with $1<\alpha<2$ in the infrared $(k \rightarrow 0)$ approximation.

Let us note that (18) has a scale $l_{0}$ :

$$
l_{0}^{-1} \equiv|2 \Gamma(-\alpha) \cos (\pi \alpha / 2) / \zeta(\alpha-1)|^{1 /(2-\alpha)}
$$

such that nontrivial expression $\mathcal{T}_{\alpha}(k) \sim|k|^{\alpha}$ appears for $k \ll k_{0}$. In the following, we consider the case $k \ll k_{0}$, i.e.

$$
\hat{\mathcal{T}}_{\alpha}(x) \sim a_{\alpha} \frac{\partial^{2}}{\partial|x|^{\alpha}} .
$$

The expression (8) for $T_{\alpha}(k)$ maps the system (2) for large number of oscillators into the continuous medium equation (17) in the limit (5). The approximate expression $\mathcal{T}_{\alpha}(k)$ (18) for $T_{\alpha}(k)$ is used to construct an operator $\hat{\mathcal{T}}_{\alpha}(x)$ that stands instead of $\hat{T}_{\alpha}$ in (4) and that appears in the final approximate equation (20). We will call $\hat{\mathcal{T}}_{\alpha}(x)$ the transform operator. The reason for that is that it can be used for in more broad sense than just for the Hamiltonian (11). Here are few examples.

The dynamical equations can be gain the term of the type (3) in complex dispersive media [26, 35, 31]. The corresponding approximate equations with fractional derivatives appear for tracer dynamics in the presence of convective rolls [41], and for the equation for surface wave interaction in ocean [42]. A specific type of equations is widely used for the oscillatory media:

$$
\frac{d}{d t} z_{n}(t)=F\left(z_{n}\right)+g_{0} \sum_{\substack{m=-\infty \\ m \neq n}}^{+\infty} J_{\alpha}(n-m)\left(z_{n}-z_{m}\right),
$$

where $z_{n}$ is the position of the $n$th oscillator in the complex plane, and $F$ is a force. As an example, for the oscillators with a limit cycle, $F$ can be taken as

$$
F(z)=(1+i a) z-(1+i b)|z|^{2} z .
$$


The nonlocal interaction is given by the power function

$$
J_{\alpha}(n)=|n|^{-\alpha-1}
$$

Similarly to (44). Using the notations (18) and (19), we can rewrite the approximate equation with fractional derivative in the form

$$
\frac{\partial Z(x, t)}{\partial t}=F(Z(x, t))+g_{0} \hat{\mathcal{T}}_{\alpha}(x) Z(x, t), \quad(1<\alpha<2),
$$

where we replace $z_{n}(t) \rightarrow Z(x, t)$ and consider $k \rightarrow 0$.

Using, for example $F(z)=0$ and expression (22) for $\hat{\mathcal{T}}_{\alpha}(x)$, we arrive to the fractional kinetic equation:

$$
\frac{\partial}{\partial t} Z(x, t)=g_{0} a_{\alpha} \frac{\partial^{\alpha}}{\partial|x|^{\alpha}} Z(x, t), \quad(1<\alpha<2)
$$

that describes the fractional superdiffusion [3, 31, 4]. For $F(z)$ in the form (24), we obtain fractional Ginzburg-Landau equation suggested in [38, 39, 40] that will be considered in the next section.

Finally, consider

$$
H_{\text {int }}=\frac{1}{2} g_{0} \sum_{\substack{n, m=-\infty \\ n \neq m}}^{+\infty} \frac{1}{|n-m|^{1+\alpha}} g\left(u_{n}\right) g\left(u_{m}\right),
$$

where $g(u)$ is some function of $u$, instead of (3). The corresponding generalization of (20) is easily obtained as

$$
\frac{\partial^{2} u(x, t)}{\partial t^{2}}=V^{\prime}(u(x, t))+g_{0} g^{\prime}(u(x, t)) \hat{\mathcal{T}}_{\alpha}(x) g(u(x, t)), \quad k \rightarrow 0, \quad(1<\alpha<2),
$$

where $g^{\prime}(u)=\partial g(u) / \partial u$ and $\hat{\mathcal{T}}_{\alpha}(x)$ is the same as in (19) or (22).

\section{Fractional Ginzburg-Landau (FGL) equation}

The one-dimensional lattice of weakly coupled nonlinear oscillators is described by

$$
\frac{d}{d t} z_{n}(t)=(1+i a) z_{n}-(1+i b)\left|z_{n}\right|^{2} z_{n}+\left(c_{1}+i c_{2}\right)\left(z_{n+1}-2 z_{n}+z_{n-1}\right)
$$

where we assume that all oscillators have the same parameters. A transition to the continuous medium assumes [8] that the difference $z_{n+1}-z_{n}$ is of order $\Delta x$, and the interaction constants $c_{1}$ and $c_{2}$ are large. Setting $c_{1}=g(\Delta x)^{-2}$, and $c_{2}=g c(\Delta x)^{-2}$, we get

$$
\frac{\partial}{\partial t} Z=(1+i a) Z-(1+i b)|Z|^{2} Z+g(1+i c) \frac{\partial^{2}}{\partial x^{2}} Z
$$


which is a complex time-dependent Ginzburg-Landau equation. The simplest coherent structures for this equation are plane-wave solutions [8]:

$$
Z(x, t)=R(K) \exp \left[i K x-i \omega(K) t+\theta_{0}\right]
$$

where

$$
R(K)=\left(1-g K^{2}\right)^{1 / 2}, \quad \omega(K)=(b-a)+(c-b) g K^{2},
$$

and $\theta_{0}$ is an arbitrary constant phase. These solutions exist for $g K^{2}<1$. Solution (32) can be interpreted as a synchronized state [8].

Let us come back to the equation for nonlinear oscillators (23) with $F(z)$ in Eq. (24) and long-range coupling (25):

$$
\frac{d}{d t} z_{n}=(1+i a) z_{n}-(1+i b)\left|z_{n}\right|^{2} z_{n}+g_{0} \sum_{m \neq n} \frac{1}{|n-m|^{\alpha+1}}\left(z_{n}-z_{m}\right)
$$

where $z_{n}=z_{n}(t)$ is the position of the $n$th oscillator in the complex plane, $1<\alpha<2$. The corresponding equation in the continuous limit and infrared approximation can be obtained in the same way as (20):

$$
\frac{\partial}{\partial t} Z=(1+i a) Z-(1+i b)|Z|^{2} Z+g(1+i c) \frac{\partial^{\alpha}}{\partial|x|^{\alpha}} Z
$$

where $g(1+i c)=g_{0} a_{\alpha}$, and $1<\alpha<2$. Eq. (35) is a fractional generalization of complex timedependent Ginzburg-Landau equation (31) (compare to (201)). Here, this equation is derived in a specific approximation for the oscillatory medium.

We seek a particular solution of (35) in the form

$$
Z(x, t)=A(K, t) e^{i K x}
$$

Eq. (36) represents a particular solution of (35) with a fixed wave number $K$.

Substitution of (36) into (35) gives

$$
\frac{\partial}{\partial t} A(K, t)=(1+i a) A-(1+i b)|A|^{2} A-g(1+i c)|K|^{\alpha} A .
$$

Rewriting this equation in polar coordinates

$$
A(K, t)=R(K, t) e^{i \theta(K, t)}
$$


we obtain

$$
\frac{d R}{d t}=\left(1-g|K|^{\alpha}\right) R-R^{3}, \quad \frac{d \theta}{d t}=\left(a-c g|K|^{\alpha}\right)-b R^{2} .
$$

The limit cycle here is a circle with the radius

$$
R=\left(1-g|K|^{\alpha}\right)^{1 / 2}, \quad g|K|^{\alpha}<1
$$

Solution of (39) with arbitrary initial conditions $R(K, 0)=R_{0}, \theta(K, 0)=\theta_{0}$ is

$$
\begin{gathered}
R(t)=R_{0}\left(1-g|K|^{\alpha}\right)^{1 / 2}\left(R_{0}^{2}+\left(1-g|K|^{\alpha}-R_{0}^{2}\right) e^{-2\left(1-g|K|^{\alpha}\right) t}\right)^{-1 / 2}, \\
\theta(t)=-\frac{b}{2} \ln \left[\left(1-g|K|^{\alpha}\right)^{-1}\left(R_{0}^{2}+\left(1-g|K|^{\alpha}-R_{0}^{2}\right) e^{-2 a t}\right)\right]-\omega_{\alpha}(K) t+\theta_{0},
\end{gathered}
$$

where

$$
\omega_{\alpha}(K)=(b-a)+(c-b) g|K|^{\alpha}, \quad 1-g|K|^{\alpha}>0 .
$$

This solution can be interpreted as a coherent structure in nonlinear oscillatory medium with long-range interaction.

If $R_{0}^{2}=1-g|K|^{\alpha}, g|K|^{\alpha}<1$, then Eqs. (41) and (42) give

$$
R(t)=R_{0}, \quad \theta(t)=-\omega_{\alpha}(K) t+\theta_{0} .
$$

Solution (44) means that on the limit cycle (40) the angle variable $\theta$ rotates with a constant velocity $\omega_{\alpha}(K)$. As the result, we have the plane-wave solution

$$
Z(x, t)=\left(1-g|K|^{\alpha}\right)^{1 / 2} e^{i K x-i \omega_{\alpha}(K) t+i \theta_{0}}, \quad 1-g|K|^{\alpha}>0,
$$

which can be interpreted as synchronized state of the oscillatory medium.

The plane-wave solution (45) is stable if parameters $a, b, c$ and $g$ satisfy

$$
0<1-g|K|^{\alpha}<a / b-(c / b) g|K|^{\alpha}<3\left(1-g|K|^{\alpha}\right) .
$$

Condition (46) defines the region of parameters for plane waves where the synchronization exists.

For initial amplitude that deviates from (40), i.e., $R_{0}^{2} \neq 1-g|K|^{\alpha}$, an additional phase shift occurs due to the term which is proportional to $b$ in (42). The oscillatory medium can be characterized by a single generalized phase variable. To define it, let us rewrite (39) as

$$
\frac{d}{d t} \ln R=\left(1-g|K|^{\alpha}\right)-R^{2}, \quad \frac{d}{d t} \theta=\left(a-c g|K|^{\alpha}\right)-b R^{2} .
$$


From (47), we obtain

$$
\frac{d}{d t} \phi=-\omega_{\alpha}(K)
$$

where

$$
\phi(R, \theta)=\theta-b \ln R
$$

is the generalized phase [8], and $\omega_{\alpha}(K)$ is defined by (43).

Eq. (48) means that generalized phase $\phi(R, \theta)$ rotates uniformly with constant velocity. For $g|K|^{\alpha}=(b-a) /(b-c)<1$, we have the lines of constant generalized phase. On $(R, \theta)$ plane these lines are logarithmic spirals $\theta-b \ln R=$ const. The decrease of $\alpha$ corresponds to the increase of $K$. For the case $b=0$ instead of spirals we have straight lines $\phi=\theta$.

Energy propagation can be characterized by the group velocity $v_{\alpha, g}=\partial \omega_{\alpha}(K) / \partial K$. From Eq. (43), we obtain

$$
v_{\alpha, g}=\alpha(c-b) g|K|^{\alpha-1} .
$$

For $|K|<K_{1}=(\alpha / 2)^{2-\alpha}$, we get $\left|v_{\alpha, g}\right|>\left|v_{2, g}\right|$. The phase velocity is

$$
v_{\alpha, p h}=\omega_{\alpha}(K) / K=(c-b) g|K|^{\alpha-1} .
$$

For $|K|<K_{2}=2^{\alpha-2}$, we have $\left|v_{\alpha, p h}\right|>\left|v_{2, p h}\right|$. Therefore long-range interaction decreasing as $|x|^{-(\alpha+1)}$ with $1<\alpha<2$ leads to increase the group and phase velocities for small wave numbers $(K \rightarrow 0)$. Note that the ratio $v_{\alpha, g} / v_{\alpha, p h}$ between the group and phase velocities of plane waves is equal to $\alpha$.

\section{Forced FGL equation for isochronous case}

In this section, we consider FGL equation (37) forced by a constant $E$ (the so-called forced isochronous case $(b=0)[8])$ :

$$
\frac{\partial}{\partial t} A=(1+i a) A-|A|^{2} A-g(1+i c)|K|^{\alpha} A-i E, \quad(\operatorname{Im} E=0),
$$

where $A=A(K, t)$, and we put for simplicity $b=0$, and $K$ is a fixed wave number. Our main goal will be transition to a synchronized states and its dependence on the order $\alpha$ of the long-range interaction.

Numerical solution of Eq. (52) was performed with parameters $a=1, g=1, c=70$, $E=0.9, K=0.1$, for $\alpha$ within interval $\alpha \in(1 ; 2)$. The results are presented on Fig. 1 . For 
$\alpha_{0}<\alpha<2$, where $\alpha_{0} \approx 1.51 \ldots$, the only stable solution is a stable fixed point. This is region of perfect synchronization (phase locking), where the synchronous oscillations have a constant amplitude and a constant phase shift with respect to the external force. For $\alpha<\alpha_{0}$ the global attractor for (52) is a limit cycle. Here, the motion of the forced system is quasiperiodic. For $\alpha=2$ there is a stable node. When $\alpha$ decreases, the stable mode transfers into a stable focus. At the transition point it loses stability, and a stable limit cycle appears. As the result, we have that the decrease of order $\alpha$ from 2 to 1 leads to the loss of synchronization (see Figure 1). For $\alpha=2.00$, and $\alpha=1.60$, we see that in the synchronization region all trajectories are attracted to a stable node.

In Fig. 1 , for $\alpha=2.00$, and $\alpha=1.60$, we see that in the synchronization region all trajectories are attracted to a stable node. For $\alpha=1.54, \alpha=1.52, \alpha=1.50$, we see that a stable limit cycle appears via the Hopf bifurcation. For $\alpha=1.54$, and $\alpha=1.52$, near the boundary of synchronization the fixed point is a focus. For $\alpha=1.50$, and $\alpha=1.4$, the amplitude of the limit cycle grows, and synchronization breaks down.

The oscillator medium can be characterized by a single generalized phase variable (49). We can rewrite (49) as

$$
\phi(X, Y)=\arctan (Y / X)-\frac{b}{2} \ln \left(X^{2}+Y^{2}\right),
$$

where $X$ and $Y$ are real and imaginary parts of $A(K, t)$. For $E=0$, the phase rotates uniformly

$$
\frac{d}{d t} \phi=-\omega_{\alpha}(K)=a-g c|K|^{\alpha}
$$

where $\omega_{\alpha}(K)$ is gived by (43) with $b=0$, and can be considered as a frequency of natural oscillations. For $E \neq 0$, Eqs. (52) and (53) give

$$
\frac{d}{d t} \phi=-\omega_{\alpha}(K)-E \cos \phi
$$

This equation has an integral of motion. The integral is

$$
I=2\left|\omega^{2}-E^{2}\right|^{-1 / 2} \arctan \left(\operatorname{sgn}(\omega-E)\left|\frac{\omega-E}{\omega+E}\right|^{-1 / 2} \tan (\phi(t) / 2)\right)+t .
$$

These expressions help to obtain the solution in form (38) for forced case (52) keeping the same notations as in (38). For polar coordinates we get

$$
\frac{d R}{d t}=\left(1-g|K|^{\alpha}\right) R-R^{3}-E \sin \theta, \quad \frac{d \theta}{d t}=\left(a-c g|K|^{\alpha}\right)-\frac{E \cos \theta}{R} .
$$



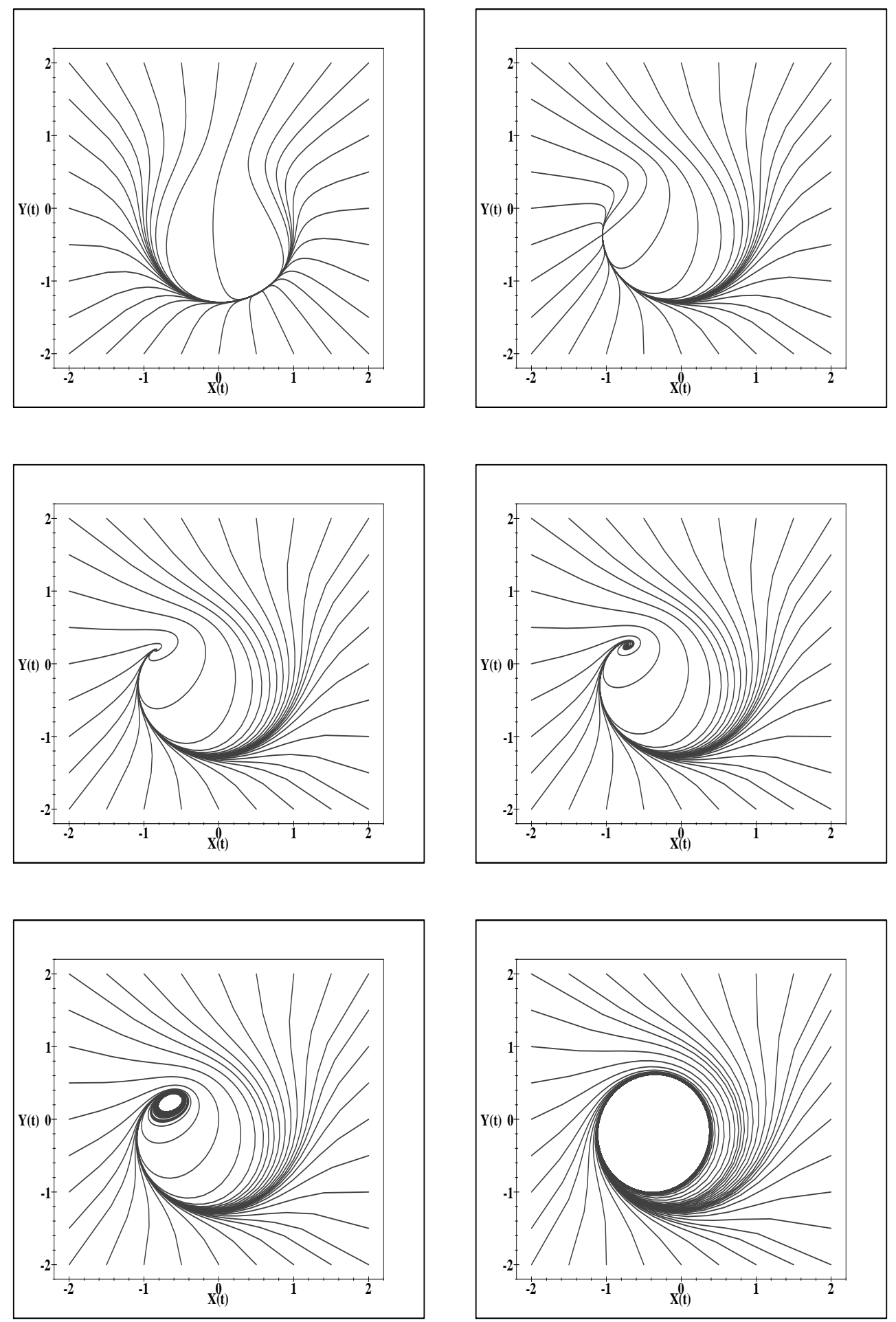

Figure 1: Approaching to the bifurcation point $\alpha=\alpha_{0}=1.51 \ldots$ and transformation to the limit cycle of solution of forced FGL equation for isochronous case with fixed wave number $K=0.1$ is represented by real $X(K, t)$ and imaginary $Y(K, t)$ parts of $A(K, t)$. The plots for orders $\alpha=2.00, \alpha=1.60, \alpha$ ₹21.54, $\alpha=1.52, \alpha=1.50, \alpha=1.40$. 
Numerical solution of (57) was performed with the same parameters as for Eq. (52), i.e., $a=1$, $g=1, c=70, E=0.9, K=0.1$, and $\alpha$ within interval $\alpha \in(1,2)$. The results are presented in Figs. 2 and 3.

The time evolition of phase $\theta(K, t)$ is given in Fig. 2 for $\alpha=2.00, \alpha=1.50, \alpha=1.47$, $\alpha=1.44, \alpha=1.40, \alpha=1.30, \alpha=1.20, \alpha=1.10$. The decrease of $\alpha$ from 2 to 1 leads to the oscillations of the phase $\theta(K, t)$ after the Hopf bifurcation at $\alpha_{0}=1.51 \ldots$, then the amplitude of phase oscillation decreases and the velocity of phase rotations increases.

The amplitude $R(K, t)$ is shown on Fig. 3 for $\alpha=1.6, \alpha=1.55, \alpha=1.55, \alpha=1.51$, $\alpha=1.50, \alpha=1.45, \alpha=1.2$. The appearance of oscillations in the plots means the loss of synchronization.

\section{Space-structures from FGL equation}

In previous sections, we considered mainly time-evolution and "time-structures" as solutions for the FGL equation. Particularly, synchronization process was an example of the solution that converged to a time-coherent structure. Here, we focuse on the space structures for the solution of FGL equation (35) with $b=c=0$ and the constants $a_{1}$ and $a_{2}$ ahead of linear term

$$
\frac{\partial}{\partial t} Z=\left(a_{1}+i a_{2}\right) Z-|Z|^{2} Z+g \frac{\partial^{\alpha}}{\partial|x|^{\alpha}} Z
$$

Let us seek a particular solution of (58) in the form

$$
Z(x, t)=R(x, t) e^{i \theta(t)}, \quad R^{*}(x, t)=R(x, t), \quad \theta^{*}(t)=\theta(t) .
$$

Substitution of (59) into (158) gives

$$
\frac{\partial}{\partial t} R=a_{1} R-R^{3}-g \frac{\partial^{\alpha}}{\partial|x|^{\alpha}} R, \quad \frac{\partial}{\partial t} \theta(t)=a_{2} .
$$

Using $\theta(t)=a_{2} t+\theta(0)$, we arrive to the existence of a limit cycle with $R_{0}=a_{1}^{1 / 2}$.

A particular solution of (60) in the vicinity of the limit cycle can be found as an expansion

$$
R(x, t)=R_{0}+\varepsilon R_{1}+\varepsilon^{2} R_{2}+\ldots, \quad(\varepsilon \ll 1) .
$$

Zero approximation $R_{0}=a_{1}^{1 / 2}$ satisfies (60) since $\partial^{\alpha} / \partial|x|^{\alpha} 1=0$, and for $R_{1}=R_{1}(x, t)$, we have

$$
\frac{\partial}{\partial t} R_{1}=-2 a_{1} R_{1}+g \frac{\partial^{\alpha}}{\partial|x|^{\alpha}} R_{1}
$$




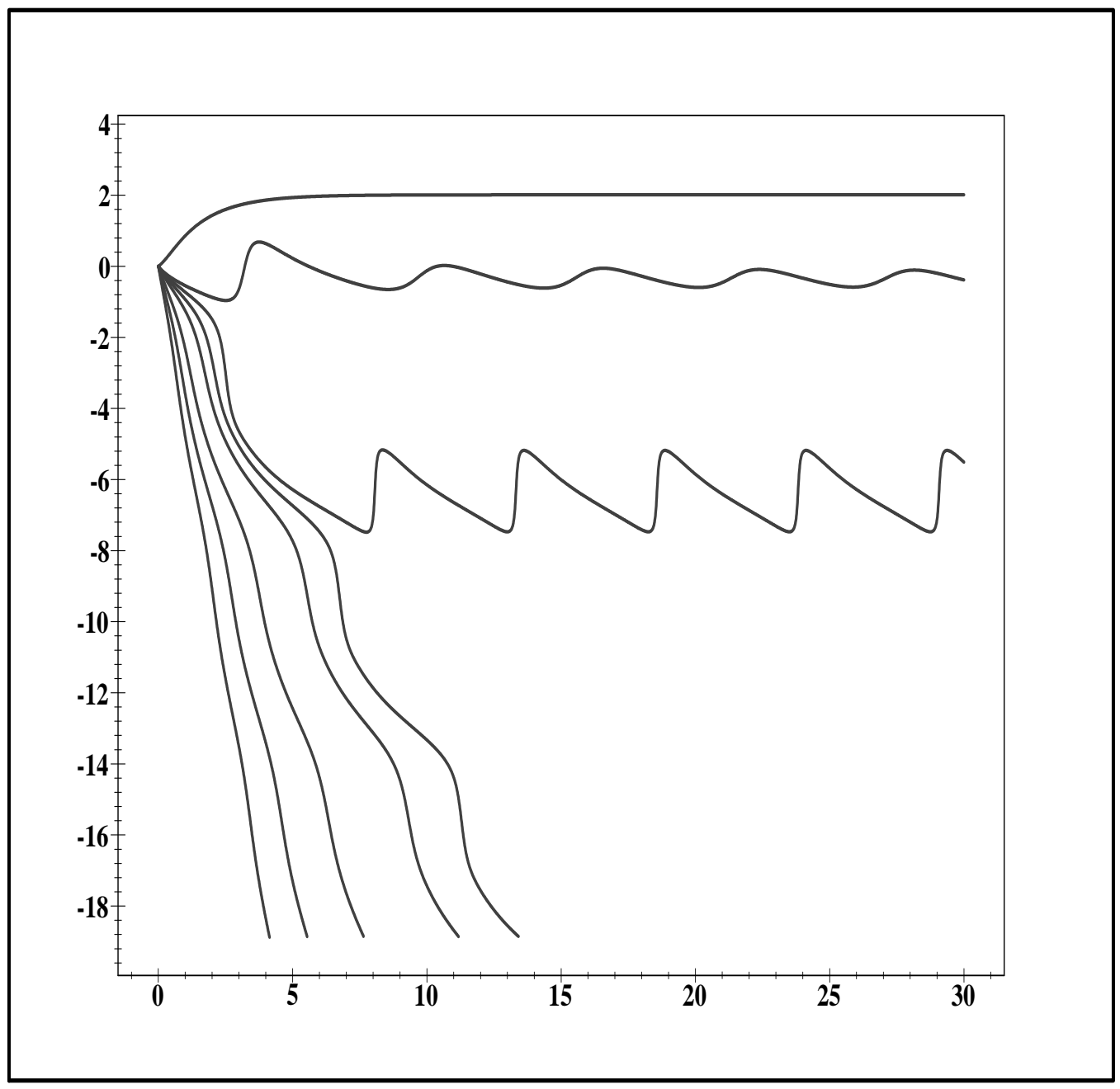

Figure 2: Phase $\theta(K, t)$ for $K=0.1$ and $\alpha=2.00, \alpha=1.50, \alpha=1.47, \alpha=1.44$, $\alpha=1.40, \alpha=1.30, \alpha=1.20, \alpha=1.10$. The decrease of order $\alpha$ corresponds to the clockwise rotation of curves. For upper curve $\alpha=2$. For the most vertical curve $\alpha=1.1$. 

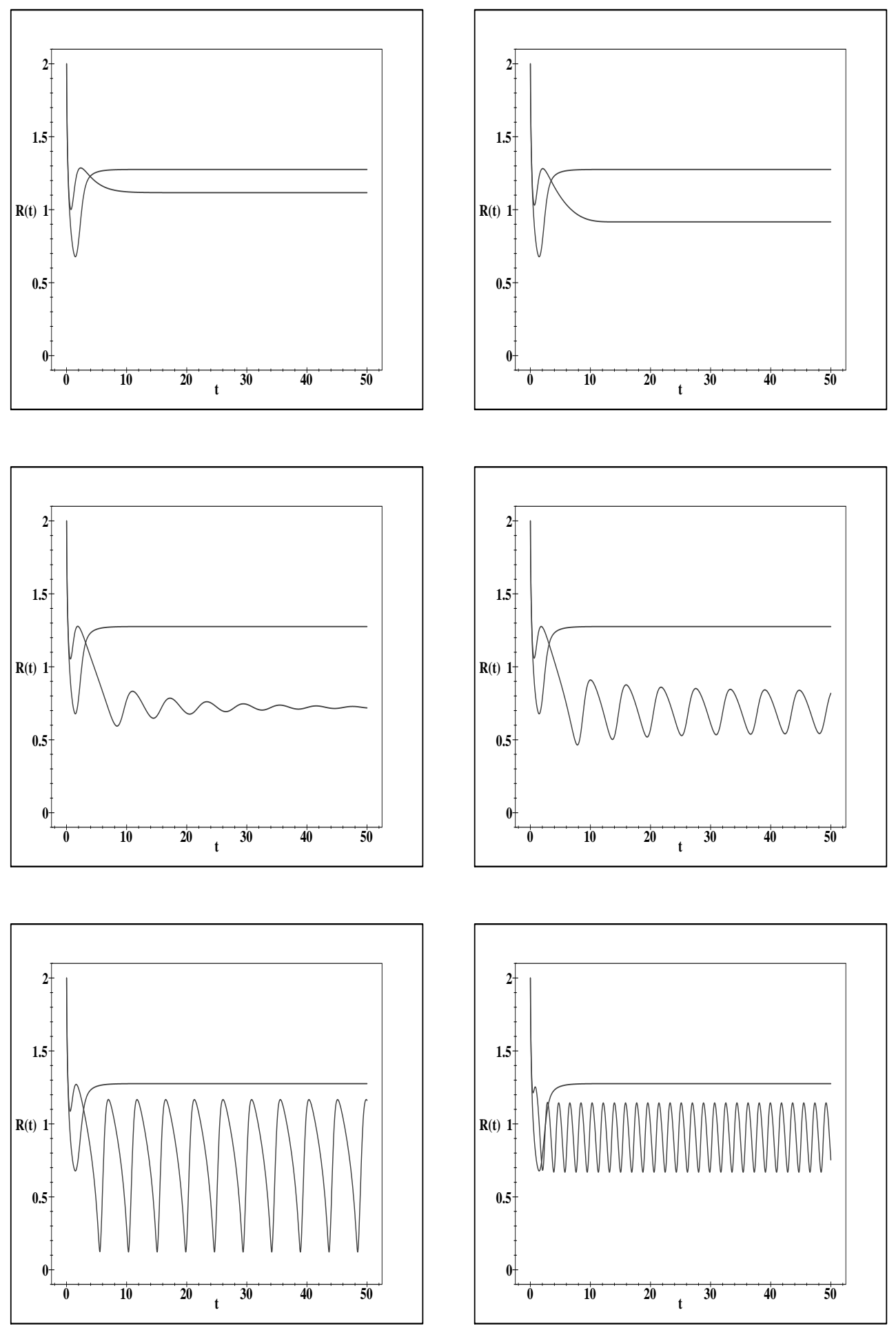

Figure 3: Amplitude $R(K, t)$. The upper curve corresponds to $\alpha=2$ for all plots. The lower curves correspond to $\alpha=1.6, \alpha=1.55, \alpha=1.51, \alpha=1.50, \alpha=1.45, \alpha=1.2$. The appearance of oscillations on the plots means the loss of synchronization. 
Consider the Cauchy problem for (62) with initial condition $R_{1}(x, 0)=\varphi(x)$, and the Green function $G(x, t)$ such that

$$
R_{1}(x, t)=\int_{-\infty}^{+\infty} G\left(x^{\prime}, t\right) \varphi\left(x-x^{\prime}\right) d x^{\prime}
$$

Let us apply Laplace transform for $t$ and Fourier transform for $x$

$$
\tilde{G}(k, s)=\int_{0}^{\infty} d t \int_{-\infty}^{+\infty} d x e^{-s t+i k x} G(x, t)
$$

Applying (64) to (62), we obtain

$$
\tilde{G}(k, s)=\frac{1}{s+2 a_{1}+g|k|^{\alpha}} .
$$

Let us first invert the Laplace transform in (65). Then, the Fourier transform of the Green function:

$$
\hat{G}(k, t)=\int_{-\infty}^{+\infty} d x e^{i k x} G(x, t)=e^{-\left(2 a_{1}+g|k|^{\alpha}\right) t}=e^{-2 a_{1} t} e^{-g|k|^{\alpha} t} .
$$

As the result, we get

$$
G(x, t)=(g t)^{-1 / \alpha} e^{-2 a_{1} t} L_{\alpha}\left(x(g t)^{-1 / \alpha}\right) .
$$

where

$$
L_{\alpha}(x)=\frac{1}{2 \pi} \int_{-\infty}^{+\infty} d k e^{-i k x} e^{-a|k|^{\alpha}}
$$

is the Levy stable p.d.f. [47].

As an example, for $\alpha=1$ we have the Cauchy distribution with respect to coordinate

$$
G(x, t)=\frac{1}{\pi} \frac{(g t)^{-1} e^{-2 a_{1} t}}{x^{2}(g t)^{-2}+1}
$$

For $\alpha=2$, we get the Gauss distribution:

$$
G(x, t)=(g t)^{-1 / 2} e^{-2 a_{1} t} \frac{1}{2 \sqrt{\pi}} e^{-x^{2} /(4 g t)} .
$$

For $1<\alpha \leq 2$ the function $L_{\alpha}(x)$ can be presented as the convergent expansion

$$
L_{\alpha}(x)=-\frac{1}{\pi x} \sum_{n=1}^{\infty}(-x)^{n} \frac{\Gamma(1+n / \alpha)}{n !} \sin (n \pi / 2) .
$$

The asymptotic $(x \rightarrow \infty, 1<\alpha<2)$ is given by

$$
L_{\alpha}(x) \sim-\frac{1}{\pi x} \sum_{n=1}^{\infty}(-1)^{n} x^{-n \alpha} \frac{\Gamma(1+n \alpha)}{n !} \sin (n \pi / 2), \quad x \rightarrow \infty,
$$


with the leading term: $L_{\alpha}(x) \sim \pi^{-1} \Gamma(1+\alpha) x^{-\alpha-1},(x \rightarrow \infty)$.

As the result, the solution of (58) is

$$
Z(x, t)=e^{i\left(a_{2} t+\theta(0)\right)}\left(a_{1}^{1 / 2}+\varepsilon(g t)^{-1 / \alpha} e^{-2 a_{1} t} \int_{-\infty}^{+\infty} L_{\alpha}\left(x^{\prime}(g t)^{-1 / \alpha}\right) \varphi\left(x-x^{\prime}\right) d x^{\prime}+O\left(\varepsilon^{2}\right)\right) .
$$

This solution can be considered as a space-time synchronization in the oscillatory medium with long-range interaction decreasing as $|x|^{-(\alpha+1)}$.

For $\varphi(x)=\delta\left(x-x_{0}\right)$, solution (73) has the form

$$
Z(x, t)=e^{i\left(a_{2} t+\theta(0)\right)}\left(a_{1}^{1 / 2}+\varepsilon(g t)^{-1 / \alpha} e^{-2 a_{1} t} L_{\alpha}\left(\left(x-x_{0}\right)(g t)^{-1 / \alpha}\right)+O\left(\varepsilon^{2}\right)\right),
$$

and the asymptotic is

$$
Z(x, t)=e^{i\left(a_{2} t+\theta(0)\right)}\left(a_{1}^{1 / 2}+\varepsilon g t e^{-2 a_{1} t} \pi^{-1} \Gamma(1+\alpha)\left(x-x_{0}\right)^{-\alpha-1}+O\left(\varepsilon^{2}\right)\right), \quad x \rightarrow \infty .
$$

This solution shows that the long-wave modes approach the limit cycle exponentially with time. For $t=1 /\left(2 a_{1}\right)$, we have the maximum of $|Z(x, t)|$ with respect to time:

$$
\max _{t>0}|Z(x, t)|=a_{1}^{1 / 2}+\varepsilon g \frac{\Gamma(1+\alpha)}{2 \pi e}\left(x-x_{0}\right)^{-\alpha-1}+O\left(\varepsilon^{2}\right) .
$$

As the result, we have the power law decay with respect to the coordinate for the space structures near the limit cycle $|Z|=a_{1}^{1 / 2}$.

\section{Conclusion}

One-dimensional chain of interacting objects, say oscillators, can be considered as a benchmark for numerous applications in physics, chemistry, biology, etc. All considered models were related mainly to the oscillating objects with long-range power wise interaction, i.e., with forces proportional to $1 /|n-m|^{s}$ and $2<s<3$. A remarkable feature of this interaction is a possibility to replace the set of coupled individual oscillator equations into the continuous medium equation with fractional space derivative of order $\alpha=s-1$, where $0<\alpha<2, \alpha \neq 1$. Such transformation is an approximation and it appears in the infrared limit for wave number $k \rightarrow 0$. This limit helps to consider different models and related phenomena in a unified way applying different tools of fractional calculus. 
A nontrivial example of general property of fractional linear equation is its solution with a power wise decay along the space coordinate. From the physical point of view that means a new type of space structures or coherent structures. The scheme of equations with fractional derivatives includes either effect of synchronization [8], breathers [48, 49, fractional kinetics [1], and others.

Discrete breathers are periodic space localized oscillations that arise in discrete and continuous nonlinear systems. Their existence was proved in Ref. [51]. Discrete breathers have been widely studied in systems with short-range interactions (for a review, see [52, 48]). Energy and decay properties of discrete breathers in systems with long-range interactions have also been studied in the framework of the Klein-Gordon [49, 53, and the discrete nonlinear Schrödinger equations [54]. Therefore, it is interesting to consider breathers solution in systems with long-range interactions in infrared approximation.

We also assume that the suggested replacement of the equations of interacting oscillators by the continuous medium equation can be used for improvement of simulations for equations with fractional derivatives.

\section{Acknowledgments}

We are thankful to N. Laskin for useful discussions and comments. This work was supported by the Office of Naval Research, Grant No. N00014-02-1-0056, the U.S. Department of Energy Grant No. DE-FG02-92ER54184, and the NSF Grant No. DMS-0417800. V.E.T. thanks the Courant Institute of Mathematical Sciences for support and kind hospitality.

\section{References}

[1] G.M. Zaslavsky, Phys. Rep. 371 (2002) 461-580.

[2] E.W. Montroll, M.F. Shlesinger, In: Studies in Statistical Mechanics, Vol. 11. J. Lebowitz, E. Montroll (Eds.), (North-Holland, Amsterdam, 1984) pp.1-121.

[3] A.I. Saichev, G.M. Zaslavsky, Chaos 7 (1997) 753-764.

[4] V.V. Uchaikin, Physics-Uspekhi 46 (2003) 821-849; J. Exper. Theor. Phys. 97 (2003) 810825. 
[5] M.M. Meerschaert, H.P. Scheffler, Limit Theorems for Sums of Independent Random Vectors: Heavy Tails in Theory and Practice (Wiley, New York, 2000)

[6] M.M. Meerschaert, D.A. Benson, B. Baeumer, Phys. Rev. E 63 (2001) 021112; Phys. Rev. E 59 (1999) 5026-5028.

[7] V. Afraimovich, A. Cordonet, N. F. Rulkov, Phys. Rev. E. 66 (2002) 016208;

V. Afraimovich, J.R. Chazottes, A. Cordonet, Discrete and Contin. Dyn. Syst. B 1 (2001) 421-442;

N.F. Rulkov, V.S. Afraimovich, C.T. Lewis, J.R. Chazottes, A. Cordonet, Phys. Rev. E 64 (2001) 016217.

[8] A. Pikovsky, M. Rosenblum, J. Kurths, Synchronization. A Universal Concept in Nonlinear Sciences (Cambridge Uviversity Press, Cambridge, 2001)

[9] S. Boccaletti, J. Kurths, G. Osipov, D.L. Valladares, C.S. Zhou, Phys. Rep. 366 (2002) $1-101$.

[10] A.T. Winfree, J. Theor. Biol. 16 (1967) 15-42.

[11] Y. Kuramoto, Chemical Oscillations, Waves, and Turbulence (Springer, Berlin, 1984)

[12] B.P. Belousov, A periodic reaction and its mechanism. in Collection of Short Papers on Radiation Medicine (Medgiz, Moscow, 1959) pp.145-152.

[13] A.M. Zhabotinsky, Proc. Acad. Sci. USSR, 157 (1964) 392-395.

[14] D. Tanaka, Y. Kuramoto, Phys. Rev. E 68 (2003) 026219.

[15] V. Casagrande, A.S. Mikhailov, Physica D 205 (2005) 154-169.

[16] S. Shima, Y. Kuramoto, Phys. Rev. E 69 (2004) 036213.

[17] Y. Kuramoto, D. Battogtokh, Nonlin. Phenom Compl. Syst. 5 (2002) 380-385.

[18] F.J. Dyson, Commun. Math. Phys. 12 (1969) 91-107;

F.J. Dyson, Commun. Math. Phys. 12 (1969) 212-215;

F.J. Dyson, Commun. Math. Phys. 21 (1971) 269-283.

[19] G.S. Joyce, J. Physics 2 (1969) 1531.

[20] J. Frohlich, R. Israel, E.H. Lieb, B. Simon, Commum. Math. Phys. 62 (1978) 1-34.

[21] H. Nakano, M. Takahashi, J. Phys. Soc. Japan 63 (1994) 926-933;

H. Nakano, M. Takahashi, Phys. Rev. B 50 (1994) 10331-10334;

H. Nakano, M. Takahashi, Phys. Rev. B 52 (1995) 6606-6610.

[22] J.R. Sousa, Eur. Phys. J. B 43 (2005) 93-96. 
[23] N. Laskin, G.M. Zaslavsky, Physica A 368 (2006) 38-54. (arXiv:nlin/0512010).

[24] R. Hilfer (Ed.), Applications of Fractional Calculus in Physics (World Scientific, Singapore, 2000)

[25] M. Caputo, Elasticita e Dissipazione (Zanichelli, Bologna, 1969)

[26] R.R. Nigmatullin, Phys. Status Solidi B 133 (1986) 425-430;

R.R. Nigmatullin, Theor. Math. Phys. 90 (1992) 242-251.

[27] A. Le Mehaute, R.R. Nigmatullin, L. Nivanen, Fleches du temps et geometric fractale (Hermes, Paris, 1998)

[28] N. Laskin, Phys. Rev. E 66 (2002) 056108;

N. Laskin, Chaos 10 (2000) 780-790;

N. Laskin, Phys. Rev. E 62 (2000) 3135-3145;

N. Laskin, Phys. Lett. A 268 (2000) 298-305.

[29] M. Naber, J. Math. Phys. 45 (2004) 3339-3352. (arXiv:math-ph/0410028)

[30] J.P. Krisch, J. Math. Phys. 46 (2005) 042506.

[31] G.M. Zaslavsky, Physica D 76 (1994) 110-122.

[32] G.M. Zaslavsky, M.A. Edelman, Physica D 193 (2004) 128-147.

[33] B.A. Carreras, V.E. Lynch, G.M. Zaslavsky, Physics of Plasmas 8 (2001) 5096-5103.

[34] V.E. Tarasov, Physics of Plasmas 12 (2005) 082106;

V.E. Tarasov, Ann. Phys. 318 (2005) 286-307;

V.E. Tarasov, Phys. Lett. A 336 (2005) 167-174;

V.E. Tarasov, Phys. Lett. A 341 (2005) 467-472;

V.E. Tarasov, Chaos 15 (2005) 023102.

[35] V.V. Zosimov, L.M. Lyamshev, Uspekni Fizicheskih Nauk 165 (1995) 361-402.

[36] F. Mainardi, R. Gorenflo, J. Comput. Appl. Math. 118 (2000) 283-299.

[37] R. Metzler, J. Klafter, Phys. Rep. 339 (2000) 1-77.

[38] H. Weitzner, G.M. Zaslavsky, Commun. Nonlin. Sci. and Numer. Simul. 8 (2003) 273-281. (nlin.CD/0212024).

[39] V.E. Tarasov, G.M. Zaslavsky, Physica A 354 (2005) 249-261.

[40] A.V. Milovanov, J.J. Rasmussen, Phys. Lett. A 337 (2005) 75-80. (arXiv:cond-mat/0309577)

[41] W.Young, A.Pumir, Y.Pomeau, Physics of Fluids A 1 (1989) 462-469. 
[42] A.J. Majda, D.W. McLaughlin, E.G. Tabak, J. Nonlin. Sciences 7 (1997) 9-44.

[43] S.G. Samko, A.A. Kilbas, O.I. Marichev, Fractional Integrals and Derivatives Theory and Applications (Gordon and Breach, New York, 1993)

[44] K.B. Oldham, J. Spanier, The Fractional Calculus (Academic Press, New York, 1974)

[45] I. Podlubny, Fractional Differential Equations (Academic Press, New York, 1999)

[46] A. Erdèlyi, W. Magnus, F. Oberhettinger, F.G. Tricomi, Higher Transcendental Functions Vol. 1. (Krieger, New York, 1981) pp.30-31.

[47] V. Feller, An Introduction to Probability Theory and its Applications Vol. 2. (Wiley, New York, 1971)

[48] S. Flach, C.R. Willis, Phys. Rep. 295 (1998) 181-264.

[49] S. Flach, Phys. Rev. E 58 (1998) R4116-R4119.

[50] O.M. Braun, Y.S. Kivshar, Phys. Rep. 306 (1998) 2-108.

[51] R.S. MacKay, S. Aubry, Nonlinearity 7 (1994) 1623-1643.

[52] S. Aubry, Physica D 103 (1997) 201-250.

[53] C. Baesens, R.S. MacKay, Helv. Phys. Acta 72 (1999) 23-32.

[54] Yu.B. Gaididei, S.F. Mingaleev, P.L. Christiansen, K.O. Rasmussen, Phys. Rev. E 55 (1997) 6141-6150. 